\title{
Negligible Effect of Ginkgo Biloba Extract on the Pharmacokinetics of Cilostazol
}

\author{
Hye Jin CHung ${ }^{1}$, Nam Sun $\mathrm{KIm}^{2}$, Eun-Jeong Kım ${ }^{3}$, Tae Kon Kım ${ }^{3}$, Keun Ho Ryu ${ }^{3}$, \\ Bong-Yong $\mathrm{LEE}^{3}$, Dong-Hyun $\mathrm{KIm}^{2}$, Changbae $\mathrm{JIN}^{2}$, and Hye Hyun $\mathrm{Yoo}^{2, *}$ \\ ${ }^{1}$ Center for Chemoinformatics Research, Korea Institute of Science and Technology, ${ }^{2}$ Doping Control Center, Korea Institute of Science \\ and Technology, Seoul 130-650, ${ }^{3}$ Life Science R\&D Center, SK Chemicals, Suwon 440-300, Republic of Korea
}

(Received April 22, 2009; Revised June 9, 2009; Accepted June 16, 2009)

\begin{abstract}
Ginkgo biloba (G. biloba) extract is a widely used phytomedicine for the oral treatment of peripheral vascular disease. Cilostazol is a synthetic antiplatelet and vasodilating agent for the treatment of intermittent claudication resulting from peripheral arterial disease. It is likely to use concomitantly G. biloba extract and cilostazol for the treatment of peripheral arterial disease, which raises a concern of increasing their adverse effects of herbal-drug interactions. To clarify any possible herbal-drug interaction between $G$. biloba extract and cilostazol, the effect of the G. biloba extract on the pharmacokinetics of cilostazol was investigated. As cilostazol is known to be eliminated mainly by cytochrome P450 (CYP)-mediated metabolism, we investigated the effects of G. biloba extract on the human CYP enzyme activities and the effect of G. biloba extract on the pharmacokinetics of cilostazol after co-administration of the two agents to male beagle dogs. The G. biloba extract inhibited more or less CYP2C8, CYP2C9, and CYP2C19 enzyme activities in the in vitro microsomal study with $\mathrm{IC}_{50}$ values of $30.8,60.5$, and $25.2 \mu \mathrm{g} / \mathrm{ml}$, respectively. In the pharmacokinetic study, co-administration with the G. biloba extract had no significant effect on the pharmacokinetics of cilostazol in dogs, although CYP2C has been reported to be responsible for the metabolism of cilostazol. In conclusion, these results suggest that there may not be a pharmacokinetic interaction between $G$. biloba extract and cilostazol.
\end{abstract}

Keywords: Ginkgo biloba extract, Cilostazol, Cytochrome P450, Pharmacokinetic interaction, Dogs

\section{INTRODUCTION}

A standardized Ginkgo biloba (G. biloba) extract, which is a phytomedicine for the oral treatment of senile dementia and peripheral vascular disease, is one of the most frequently prescribed phytomedicines in Europe and Asia, and one of the most widely sold herbal dietary supplements in the United States (Blumenthal et al., 1998; Blumenthal, 2001). G. biloba extract is known to contain ginkgo flavone glycosides, terpene lactones, and proanthocyanidins (Chan et al., 2007). Although the mechanism of the action is not clear, it is generally agreed that it protects against oxidative cell damage from free radicals (Sastre et al., 1998), improves blood flow, including microcirculation in small capillaries, to most organs (Jung et al.,

${ }^{*}$ Corresponding author

Tel: +82-2-958-6422 Fax: +82-2-958-6677

E-mail: behappy@kist.re.kr
1990), and blocks many of the effects of the platelet-activating factor that have been related to the development of a number of cardiovascular, renal, respiratory, and CNS disorders (Oberpichler et al., 1990).

Cilostazol is a synthetic antiplatelet and vasodilating agent for the treatment of intermittent cloudication resulting from peripheral arterial disease. Cilostazol is a selective cAMP phosphodiesterase inhibitor. It inhibits platelet aggregation and also acts as a direct arterial vasodilator (Kimura et al., 1985). Its main effects are dilation of the arteries supplying blood to the legs and decreasing platelet coagulation (Fontana and Reny, 2007).

Both antiplatelet agents of G. biloba extract and cilostazol are used to treat peripheral arterial disease and improve blood circulation. Many studies have demonstrated that the combination treatment of aniplatelet agents could be beneficial in cerebral and peripheral arterial disease (Kim et al., 1998; Aldandashi et al., 2007; Fontana and 
Reny, 2007). Moreover, G. biloba extract enhanced the antiplatelet and antithrombotic effects of cilostazol without the prolongation of bleeding time (our unpublished data). Thus, it is likely that $G$. biloba extract is prescribed in combination with cilostazol for their possible synergic effects. However, such a combination treatment may lead to herb-drug interactions. Therefore, it is necessary to evaluate the drug interaction potential between these two agents.

Cilostazol is extensively metabolized in rats, dogs, and humans (Akiyama et al., 1985b). It was reported that CYP isoforms $3 \mathrm{~A} 4,1 \mathrm{~B} 1,3 \mathrm{~A} 5,2 \mathrm{C} 19$, and $2 \mathrm{C} 8$ are involved in the metabolism of cilostazol (Hiratsuka et al., 2007). Cilostazol is known to be eliminated mainly by CYP-mediated metabolism and consequently to interact with some drugs in vitro and in vivo (Bramer and Suri 1999; Suri and Bramer 1999; Abbas et al., 2000), indicating that cilostazol carries a considerable potential for drug interactions. Co-administration of cilostazol with a CYP inhibitor (such as omeprazol, erythromycin, and quinidine) may result in an increase in the systemic exposure of cilostazol in humans and consequently cause adverse effects (Bramer and Suri, 1999; Suri and Bramer, 1999; Suri et al., 1999).

G. biloba extract is often taken in combination with prescription and conventional medications, causing herb-drug interactions (Gurley et al., 2005; Hu et al., 2005). There are controversial reports on the CYP inhibition by G. biloba extract. In some reports, ginkgolic acids, one of the components of $G$. biloba extract, were shown to be potent inhibitors of CYP1A2, CYP2C9, and CYP2C19 using CDNA expressed CYP enzymes (Zou et al., 2002). Although flavonol aglycones did show significant inhibitory activity against CYP1A2, 2C9, and $3 \mathrm{~A}$, other findings show that the most abundant components of ginkgo preparations in clinical use (terpene trilactones and flavonol glycosides) do not significantly inhibit major CYP enzymes in human liver microsomes (von Moltke et al., 2004).

As mentioned above, cilostazol is metabolized via CYP3A4, 1B1, 3A5, 2C19, and 2C8. Therefore, the pharmacokinetics of cilostazol could be affected by co-administration with $G$. biloba extract if the $G$. biloba extract possesses inhibitory activity on these CYP subtypes. Therefore, it is necessary to characterize the inhibitory activity of $G$. biloba extract on the CYP enzymes and investigate pharmacokinetic herb-drug interactions between the G. biloba extract and the cilostazol. This paper presents the effect of G. biloba extract on human CYP enzyme activity and the pharmacokinetics of cilostazol after the co-administration of these two agents to male beagle dogs.

\section{MATERIALS AND METHODS}

\section{Chemicals}

The standardized G. biloba extract, cilostazol, and 4-isopropylantipyrine were obtained from SK Chemical Co., Ltd. (Seoul, Korea). The Standardized G. biloba extract contains $22.0-27.0 \%$ ginkgo-flavonol glycosides and $5.4-12.0 \%$ terpene lactones $(2.6-5.8 \%$ bilobalide and $2.8-6.2 \%$ ginkgolides). Pooled human liver microsome, phenacetin, coumarin, paclitaxel, diclofenac, mephenytoin, dextromethorphan, midazolam, O-deethylphenacetin, 7-hydroxycoumarin, 6-hydroxypaclitaxel, 4-hydroxydiclofenac, 4-hydroxymephenytoin, O-demethyldextromethorphan, and 1-hydroxymidazolam were purchased from BD Biosciences (Woburn, MA). Glucose 6-phosphate, $\beta$-NADP ${ }^{+}$, and glucose 6-phosphate dehydrogenase were supplied by Sigma Corporation (St. Louis, MO). All other chemicals used were of analytical grade.

\section{Animals}

Male beagle dogs (weighing 9-11 kg) were purchased from Central Lab. Animal Inc. (Seoul, Republic of Korea). Dogs were kept at an environmental temperature of $23 \pm$ $2^{\circ} \mathrm{C}$ and relative humidity of $55 \pm 5 \%$ with a $12 \mathrm{~h}$ light-dark cycle. Each dog was unrestrained and individually housed in a stainless steel cage, and allowed access to food and water ad libitum. Washout periods of one week were used between studies. Animals were deprived of food for $12 \mathrm{~h}$ before dosing. The protocol of animal study was in accordance with the regulations of the Korea Institute of Science and Technology.

\section{Microsomal incubation}

Incubation studies were designed to determine the effects of G. biloba extract on various CYP isozyme activities. The incubation mixture contained $1 \mathrm{mg} / \mathrm{ml}$ microsomal protein and various CYP isoform-specific substrates in $0.2 \mathrm{ml}$ of $0.1 \mathrm{M}$ potassium phosphate buffer ( $\mathrm{pH} 7.4$ ). Reactions were initiated by the addition of the NADPH generating system (10 mM glucose 6-phosphate, $0.67 \mathrm{mM} \beta$-NADP $^{+}$, and $1 \mathrm{U} / \mathrm{ml}$ glucose-6-phosphate dehydrogenase), at various concentrations $(10,20,40,60,80$, and $100 \mu \mathrm{g} / \mathrm{ml})$ of G. biloba extract and continued for $30 \mathrm{~min}$ in a water bath at $37^{\circ} \mathrm{C}$. After the incubation, the reaction was stopped by adding $0.4 \mathrm{ml}$ of $0.1 \%$ acetic acid. The following substrate concentrations were used (Tucker et al., 2001): $40 \mu \mathrm{M}$ for phenacetin, $2.5 \mu \mathrm{M}$ for coumarin and midazolam, $10 \mu \mathrm{M}$ for paclitaxel and diclofenac, $160 \mu \mathrm{M}$ for mephenytoin, and $5 \mu \mathrm{M}$ for dextromethorphan. 


\section{CYP substrate metabolism assays}

The metabolites of the CYP isozyme specific substrate were analyzed by a liquid chromatography-tandem mass spectrometric (LC-MS/MS) method. Four microliter of $16 \mu \mathrm{M}$ terfenadine in acetonitrile was added as an internal standard (IS) to the reaction mixture. The reaction mixture was loaded to a solid-phase extraction cartridge (a preconditioned $\mathrm{C}_{18}$ Sep-pak ${ }^{\circledR}$ with methanol and deionized water). The cartridge was washed with $1 \mathrm{ml}$ of $0.1 \%$ acetic acid three times. The analytes were then eluted with $1 \mathrm{ml}$ of methanol. The methanol extract was evaporated under a gentle stream of nitrogen gas. The residue was reconstituted in a $40-\mu \mathrm{l}$ aliquot of $30 \%$ acetonitrile and a $10-\mu \mathrm{l}$ aliquot was injected directly onto a reversed-phase $\left(\mathrm{C}_{18}\right)$ HPLC column (Xterra ${ }^{\circledR}, 3.5 \mu \mathrm{m}, 2.1 \times 150 \mathrm{~mm}$ ). The LC-MS/MS system consisted of a LC-10ADvp binary pump system (Shimadzu, Japan) with an API2000 triple quadrupole mass spectrometer (Applied Biosystems-SCIEX, Concord, Canada) equipped with an electrospray ionization (ESI) source. HPLC mobile phases consisted of (A) $0.1 \%$ aqueous formic acid and (B) $90 \%$ acetonitrile in $0.1 \%$ formic acid. The initial composition was $5 \% \mathrm{~B}$, programmed linearly to $70 \% \mathrm{~B}$ after $3 \mathrm{~min}$, and held for $1 \mathrm{~min}$. The gradient was then changed back to the initial condition in $0.5 \mathrm{~min}$ and kept at the initial condition for $2 \mathrm{~min}$. The total run time was $6 \mathrm{~min}$. A gradient program was used for the HPLC separation with a flow rate of $0.2 \mathrm{ml} / \mathrm{min}$. ESI was performed in the positive mode with nitrogen as the nebulizing, turbo spray, and curtain gas, with the optimum values set at 40,75 , and 40 (arbitrary units). The multiple reaction monitoring (MRM) detection method was employed for the detection of metabolites. Transitions monitored for metabolites were $\mathrm{m} / \mathrm{z} 152 \rightarrow 110$ for O-deethylphenacetin, $\mathrm{m} / \mathrm{z} 163 \rightarrow 107$ for 7 -hydroxycoumarin, $\mathrm{m} / \mathrm{z} \quad 870 \rightarrow 286$ for 6-hydroxypaclitaxel, $\mathrm{m} / \mathrm{z} \quad 312 \rightarrow 230$ for 4-hydroxydiclofenac, $m / z 235 \rightarrow 150$ for 4-hydroxymephenytoin, $\mathrm{m} / \mathrm{z} 258$ $\rightarrow 157$ for O-demethyldextromethorphan, $\mathrm{m} / \mathrm{z} 342 \rightarrow 324$ for 1 -hydroxymidazolam, and $\mathrm{m} / \mathrm{z} 472 \rightarrow 436$ for terfenadine (IS).

\section{Data analysis}

The $\mathrm{IC}_{50}$ values for the activity-concentration curves from individual experiments were calculated with Sigma-plot software (Systat Software Inc., Chicago, IL), using a nonlinear regression method.

\section{Oral administration of cilostazol and G. biloba extract to dogs}

Six male beagle dogs were randomly divided into three groups and each group received three treatments for 3-pe- riod, in a $3 \times 3$ Latin Square Design: $10 \mathrm{mg} / \mathrm{kg}$ oral cilostazol without G. biloba extract, $10 \mathrm{mg} / \mathrm{kg}$ oral cilostazol with 8 $\mathrm{mg} / \mathrm{kg} \mathrm{G}$. biloba extract, and $10 \mathrm{mg} / \mathrm{kg}$ oral cilostazol with $16 \mathrm{mg} / \mathrm{kg}$ G. biloba extract. Each experimental period consisted of a one-day single dose treatment and a one week wash-out period. The foot cephalic vein of each dog was cannulated with sterile intravascular catheters for blood sampling.

Cilostazol at a dose of $10 \mathrm{mg} / \mathrm{kg}$ with or without G. biloba extract was orally administered to the dogs using a capsule. Co-administration groups received G. biloba extract and cilostazol simultaneously. A blood sample (approximately $1 \mathrm{ml}$ ) was collected via the cephalic vein at $0,0.5,1,2,3,4,6$, and $8 \mathrm{~h}$ after the oral administration of the drug(s). Blood samples were centrifuged immediately and a $500 \mu \mathrm{l}$ aliquot of plasma sample was stored in a $-20^{\circ} \mathrm{C}$ freezer until the LC-MS/MS analysis of the cilostazol.

\section{Analytical method of cilostazol in dog plasma}

Concentrations of cilostazol in the dog plasma samples were determined by a slight modification of the reported LC-MS/MS method (Nirogi et al., 2006). An aliquot of 20- $\mu$ I internal standard $(0.1 \mu \mathrm{g} / \mathrm{ml}$ 4-isopropylantipyrine in $50 \%$ methanol) was added to a 100- $\mu$ l aliquot of plasma sample. The mixture was then extracted with $1 \mathrm{ml}$ of extraction solvent (ethyl acetate:methylene chloride=7:3). The organic layer was transferred into a clean microcentrifuge tube and evaporated under a gentle stream of nitrogen gas. The residue was reconstituted in a $50-\mu \mathrm{l}$ aliquot of the mobile phase and a $5-\mu \mathrm{l}$ aliquot was injected directly onto a LC-MS/MS system. The mobile phase, $5 \mathrm{mM}$ ammonium formate buffer ( $\mathrm{pH} 4.0)$ :acetonitrile (90:10, $\mathrm{v} / \mathrm{v}$ ), was run at a flow-rate of $0.2 \mathrm{ml} / \mathrm{min}$. The LC-MS/MS system was the same as that used in the CYP substrate assay. The MRM detection was employed; the transitions monitored were $\mathrm{m} / \mathrm{z} 370 \rightarrow 125$ for cilostazol, $\mathrm{m} / \mathrm{z} 231 \rightarrow 56$ for 4-isopropylantipyrine (IS). Retention times of the cilostazol and IS were approximately $2.2 \mathrm{~min}$ and $2.4 \mathrm{~min}$, respectively. Calibration curves of cilostazol were linear over the ranges studied with $r^{2}>0.999$. The lower limit of quantification of the cilostazol in the dog plasma was $1 \mathrm{ng} / \mathrm{ml}$. The coefficients of variations of the cilostazol (intra-day and inter-day) were below $10.7 \%$ and the accuracy ranged from 91.7 to $113 \%$.

\section{Pharmacokinetic analysis}

The total area under the plasma concentration-time curve from time zero to time infinity (AUC) was calculated using the trapezoidal rule-extrapolation method (Chiou, 1978). The area from the last datum point to time infinity 
was estimated by dividing the last measured plasma concentration by the terminal-phase rate constant.

Standard methods (Gibaldi and Perrier, 1982) were used to calculate the pharmacokinetic parameters using a noncompartmental analysis (WinNonlin ${ }^{\circledR}$; Pharsight Corporation, Mountain View, CA). The peak plasma concentration $\left(C_{\max }\right)$ and time to reach $C_{\max }\left(T_{\max }\right)$ were directly read from the experimental data. The apparent oral clearance of cilostazol (CL/F) was calculated as dose/AUC.

Table I. $\mathrm{IC}_{50}$ of the G. biloba extract on CYP isozyme specific marker activities in pooled human liver microsomes

\begin{tabular}{cc}
\hline P450 isozyme & $\begin{array}{c}\mathrm{IC}_{50}(\mu \mathrm{g} / \mathrm{ml}) \\
\text { mean } \pm \text { S.D. }\end{array}$ \\
\hline CYP1A2 & $>100$ \\
CYP2A6 & $>100$ \\
CYP2C8 & $30.8 \pm 1.1$ \\
CYP2C9 & $60.5 \pm 8.3$ \\
CYP2C19 & $25.2 \pm 3.0$ \\
CYP2D6 & $>100$ \\
CYP3A4 & $>100$ \\
\hline
\end{tabular}

\section{Statistical analysis}

A $p$-value of less than 0.05 was considered statistically significant using a Duncan's multiple range test of the Statistical Package for the Social Sciences posteriori analysis of the repeated measure analysis of variance test. All results are expressed as mean \pm standard deviation (S.D.) except median (ranges) for $T_{\max }$.

\section{RESULTS}

\section{Microsomal incubation}

The inhibitory effects of G. biloba extract on the CYP isoform-specific activities were evaluated in human liver microsomes at various concentrations of $\mathrm{G}$. biloba extract. The $I_{50}$ values of the $G$. biloba extract for various CYP enzyme marker activities are presented in Table I. The G. biloba extract showed inhibitory activities on specific isozymes. The G. biloba extract did not inhibit the phenacetin O-deethylation (CYP1A2), coumarin 7-hydroxylation (CYP2A6), dextromethorphan O-demethylation (CYP2D6), or the midazolam 1-hydroxylation (CYP3A4) activities $\left(\mathrm{IC}_{50}>100 \mu \mathrm{g} /\right.$ $\mathrm{ml}$ ). The G. biloba extract inhibited the paclitaxel 6-hydroxylation (CYP2C8), diclofenac 4-hydroxylation (CYP2C9),

Table II. Pharmacokinetic parameters (mean \pm S.D.) of cilostazol after oral administration $(10 \mathrm{mg} / \mathrm{kg})$ of cilostazol alone or co-administration of $\mathrm{G}$. biloba extract $(8 \mathrm{mg} / \mathrm{kg}$ or $16 \mathrm{mg} / \mathrm{kg})$ to dogs $(\mathrm{n}=6)$

\begin{tabular}{lccccc}
\hline \multicolumn{1}{c}{ Parameters } & $\begin{array}{c}\text { Without G. biloba } \\
\text { extract }\end{array}$ & $\begin{array}{c}\text { With G. biloba extract } \\
(8 \mathrm{mg} / \mathrm{kg})\end{array}$ & $\begin{array}{c}\text { With G. biloba extract } \\
(16 \mathrm{mg} / \mathrm{kg})\end{array}$ & $F^{2}$ value & $p$ value \\
\hline$C_{\max }(\mathrm{ng} / \mathrm{ml})$ & $417 \pm 290$ & $418 \pm 168$ & $499 \pm 280$ & 0.177 & 0.841 \\
$T_{\max }(\mathrm{h})^{\mathrm{c}}$ & $1.5(1-4)$ & $1.5(1-3)$ & $2(1-2)$ & 0.020 & 0.980 \\
$\mathrm{t}_{1 / 2}(\mathrm{~h})$ & $2.55 \pm 1.10$ & $2.22 \pm 0.873$ & $2.29 \pm 1.25$ & 0.182 & 0.836 \\
CL/F $(\mathrm{ml} / \mathrm{min} / \mathrm{kg})$ & $131 \pm 62.7$ & $145 \pm 94.3$ & $119 \pm 45.1$ & 0.200 & 0.822 \\
AUC $(\mathrm{ng} \mathrm{h} / \mathrm{ml})$ & $1,610 \pm 972$ & $1,410 \pm 507$ & $1,670 \pm 837$ & 0.155 & 0.858 \\
\hline
\end{tabular}

${ }^{\mathrm{a}}$ There was no significant difference among the three groups, ${ }^{\mathrm{b}} 95 \%$ confidence intervals, ${ }^{\mathrm{c}} T_{\max }$ is expressed as median (ranges).
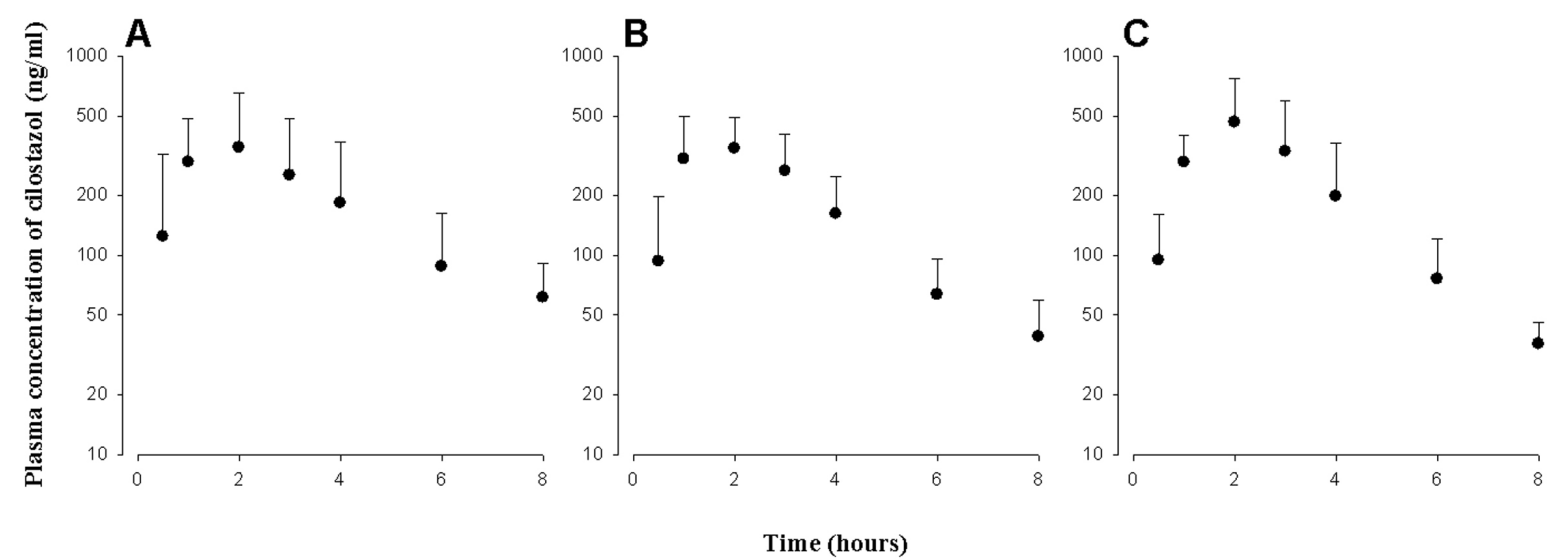

Fig. 1. Mean plasma concentration-time profiles of cilostazol after oral administration of cilostazol without G. biloba extract (A) and with G. biloba extract $(B ; 8 \mathrm{mg} / \mathrm{kg}$ and $\mathrm{C} ; 16 \mathrm{mg} / \mathrm{kg})$ to dogs $(\mathrm{n}=6)$. Bars represent standard deviation. 
and mephenytoin 4-hydroxylation (CYP2C19) activities with $\mathrm{IC}_{50}$ of $30.8,60.5$, and $25.2 \mu \mathrm{g} / \mathrm{ml}$, respectively.

\section{Pharmacokinetics of cilostazol after oral administration of cilostazol with or without administration of $G$. biloba extract to dogs}

The mean plasma cilostazol concentration-time profiles from the three treatments are shown in Fig. 1 and relevant pharmacokinetic parameters are summarized in Table II. After oral administration, the plasma concentrations of the cilostazol reached peak concentration $\left(C_{\max }\right)$ at $1-4 \mathrm{~h}$ and declined (Fig. 1) with mean terminal half-lives of 2.55, 2.22, and 2.29 hours for the cilostazol only, with $8 \mathrm{mg} / \mathrm{kg} \mathrm{G}$. biloba extract, and with $16 \mathrm{mg} / \mathrm{kg}$ G. biloba extract, respectively (Table II). Pharmacokinetic parameters were comparable in terms of $C_{\max }, T_{\max }, \mathrm{t}_{1 / 2}, \mathrm{AUC}$, and CL/F (Table II). Co-administration with the G. biloba extract had no significant effect on the pharmacokinetics of the cilostazol.

\section{DISCUSSION}

Recently, several reports have been published that combination therapies of antiplatelet agents were more effective than a single drug administration (Kim et al., 1998; Aldandashi et al., 2007; Fontana and Reny, 2007). However, for such a combination therapy, drug interactions should be considered to prevent likely side-effects caused by concomitant use of these agents. It is well known that inhibition or induction of drug metabolizing enzyme is responsible for drug interaction when one agent changes pharmacokinetic properties of a concurrently administered drug. For example, hydrocortisone significantly enhanced the bioavailability of orally administered loratadine in rats (Choi et al., 2009). Actually, it was one of the most important reasons for the early termination of the clinical trial (furafylline), the refusal of approval (mibefradil in Sweden), the withdrawal from the market (sorivudine, terfenadine, astemizole, cisapride), and the restriction of use (ketoconazole) (Tucker et al., 2001). There are also many cases of herb-drug interactions (Fugh-Berman, 2000): bleeding when warfarin is combined with ginkgo ( $G$. biloba), garlic (Allium sativum), dong qual (Angelica sinensis), or danshen (Salvia miltiorrhiza); mild serotonin syndrome in patients who mix St John's wort (Hypericum perforatum) with serotonin-reuptake inhibitors; and, decreased bioavailability of digoxin, theophylline, cyclosporin, and phenprocoumon when these drugs are combined with St John's wort.

This study was designed to determine the effect of $G$. bi- loba extract on the pharmacokinetics of cilostazol. Cilostazol is cleared mainly via the metabolic route in rats, dogs, and humans (Akiyama et al., 1985b). Several metabolites of cilostazol were identified (Akiyama et al., 1985b) and CYP3A4, 1B1, 3A5, 2C19, and 2C8 have been known to involve the formation of the major metabolites, OPC-13015 and OPC-13213 (Abbas et al., 2000). Pharmacokinetic changes of cilostazol may be possible with the co-administration of the CYP inhibitor because it is metabolized mainly via CYP. There are some reports that $G$. biloba extract inhibited in vitro CYP $3 \mathrm{~A}$ mediated metabolism (Zou et al., 2002; Hellum and Nilsen, 2008). The results from the present study (Table I) indicated that G. biloba extract mildly inhibits CYP2C8, CYP2C9, and CYP2C19 activities with $\mathrm{IC}_{50}$ of $30.8,60.5$, and $25.2 \mu \mathrm{g} / \mathrm{ml}$, respectively, in human liver microsomes. These results suggest CYP2C8, CYP2C9, or CYP2C19-mediated drug metabolism, especially the biotransformation of cilostazol by $\mathrm{CYP} 2 \mathrm{C} 8$ or CYP2C19 might be affected by G. biloba extract. Therefore, pharmacokinetic interaction between these two reagents could not be excluded and pharmacokinetic studies in dogs were followed to determine whether the pharmacokinetics of cilostazol is affected by the co-administration of G. biloba extract in vivo. The in vitro assay was carried out using human liver microsomes for the best prediction of pharmacokinetic interactions in human but the in vivo study was carried out using animals because of safety concerns. The major two canine CYP2Cs, CYP2C21 and $2 \mathrm{C} 41$, were known to have $74-83 \%$ nucleotide and $67-76 \%$ amino acid identity with the human $\mathrm{CYP} 2 \mathrm{C} 8,2 \mathrm{C} 9$, and 2C19 (Blaisdell et al., 1998) thus, canine CYP2C21 and 2C41 may play a similar role to human CYP2Cs. In our study, there was no difference in the pharmacokinetic parameters of cilostazol when cilostrazol was administered alone or with G. biloba extract and the pharmacokinetic parameters of cilostazol obtained from the present study were comparable to those previously reported (Akiyama et al., 1985a; Jinno et al., 2006). The $I_{50}$ of G. biloba extract for CYP2C8 or CYP2C19 may be much higher than its level in plasma after oral administration of 8 or $16 \mathrm{mg} / \mathrm{kg} \mathrm{G}$. biloba extract. Therefore, G. biloba extract could not actually affect the metabolism of cilostazol. Taken together, it is considered that there is no pharmacokinetic interaction between G. biloba extract and cilostazol, which is partly consistent with the previous report regarding the pharmacodynamic interaction of G. biloba with cilostazol (Aruna and Naidu, 2007). They reported that the possibility of pharmacodynamic interaction between cilostazol and G. biloba was not shown regarding an inhibitory effect of platelet aggregation although the bleeding time prolongation effect of 
cilostazol was potentiated by a co-administration with $G$. biloba.

\section{CONCLUSIONS}

The G. biloba extract mildly inhibited the CYP2C8 and CYP2C19 activities in vitro. However, co-administration of G. biloba extract with cilostazol did not alter the pharmacokinetics of cilostazol in dogs. Further studies should be followed to characterize herb-drug interactions of G. biloba extract with cilostazol in human.

\section{ACKNOWLEDGMENTS}

This study was supported in part by SK Chemicals Ltd. and in part by Seoul Research and Business Development Program.

\section{REFERENCES}

Abbas, R., Chow, C. P., Browder, N. J., Thacker, D., Bramer, S. L., Fu, C. J. and Forbes, W. (2000). In vitro metabolism and interaction of cilostazol with human hepatic cytochrome P450 isoforms. Hum. Exp. Toxicol. 19, 178-184.

Akiyama, H., Kudo, S. and Shimizu, T. (1985a). The absorption, distribution and excretion of a new antithrombotic and vasodilating agent, cilostazol, in rat, rabbit, dog and man. Arzneimittelforschung 35, 1124-1132.

Akiyama, H., Kudo, S. and Shimizu, T. (1985b). The metabolism of a new antithrombotic and vasodilating agent, cilostazol, in rat, dog and man. Arzneimittelforschung 35, 1133-1140.

Aldandashi, S., Noor, R., Wang, C. X., Uddin, G. and Shuaib, A. (2007). Combination treatment with dipyridamole, aspirin, and tPA in an embolic model of stroke in rats. Exp. Neurol. 205, 563-568.

Aruna, D. and Naidu, M. U. (2007). Pharmacodynamic interaction studies of Ginkgo biloba with cilostazol and clopidogrel in healthy human subjects. Br. J. Clin. Pharmacol. 63, 333-338.

Blaisdell, J., Goldstein, J. A. and Bai, S. A. (1998). Isolation of a new canine cytochrome P450 cDNA from the cytochrome P450 2C subfamily (CYP2C41) and evidence for polymorphic differences in its expression. Drug Metab. Dispos. 26, 278-283.

Blumenthal, M. (2001). Herb sales down $15 \%$ in mainstream market. HerbalGram. 51, 69.

Blumenthal, M., Busse, W. R., Goldberg, A., Gruenwald, J., Hall, T., Riggins, C. W. and Rister, R. S. (Eds.), (1998). The complete German commission E monographs: Therapeutic guide to herbal medicines. The American Botanical Council, Austin.

Bramer, S. L. and Suri, A. (1999). Inhibition of CYP2D6 by quinidine and its effects on the metabolism of cilostazol. Clin. Pharmacokinet. 37(Suppl 2), 41-51.

Chan, P. C., Xia, Q. and Fu, P. P. (2007). Ginkgo biloba leave extract: biological, medicinal, and toxicological effects. $J$. Environ. Sci. Health C Environ. Carcinog. Ecotoxicol. Rev. 25, 211-244.
Chiou, W. L. (1978). Critical evaluation of potential error in pharmacokinetic studies using the linear trapezoidal rule method for the calculation of the area under the plasma level-time curve. J. Pharmacokinet. Biopharm. 6, 539-549.

Choi, J. S., Choi, I. and Burm J. P. (2009). Effects of hydrocortisone on the pharmacokinetics of loratadine after oral and intravenous loratadine administration to rats. Biomol. Ther. 17, 205-210.

Fontana, P. and Reny, J. L. (2007). New antiplatelet strategies in atherothrombosis and their indications. Eur. J. Vasc. Endovasc. Surg. 34, 10-17.

Fugh-Berman, A. (2000). Herb-drug interactions. Lancet $\mathbf{3 5 5}$, 134-138.

Gibaldi, M. and Perrier, D. (1982). Pharmacokinetics. 2nd ed. Marcel-Dekker, New York.

Gurley, B. J., Gardner, S. F., Hubbard, M. A., Williams, D. K., Gentry, W. B., Cui, Y. and Ang, C. Y. (2005). Clinical assessment of effects of botanical supplementation on cytochrome P450 phenotypes in the elderly: St John's wort, garlic oil, Panax ginseng and Ginkgo biloba. Drugs Aging 22 525-539.

Hellum, B. H. and Nilsen, O. G. (2008). In vitro inhibition of CYP3A4 metabolism and p-glycoprotein-mediated transport by trade herbal products. Basic Clin. Pharmacol. Toxicol. 102, 466-475.

Hiratsuka, M., Hinai, Y., Sasaki, T., Konno, Y., Imagawa, K., Ishikawa, M. and Mizugaki, M. (2007). Characterization of human cytochrome P450 enzymes involved in the metabolism of cilostazol. Drug Metab. Dispos. 35, 1730-1732.

Hu, Z., Yang, X., Ho, P. C., Chan, S. Y., Heng, P. W., Chan, E., Duan, W., Koh, H. L. and Zhou, S. (2005). Herb-drug interactions: a literature review. Drugs 65, 1239-1282.

Jinno, J., Kamada, N., Miyake, M., Yamada, K., Mukai, T., Odomi, M., Toguchi, H., Liversidge, G. G., Higaki, K. and Kimura, T. (2006). Effect of particle size reduction on dissolution and oral absorption of a poorly water-soluble drug, cilostazol, in beagle dogs. J. Control. Release. 111, 56-64.

Jung, F., Mrowietz, C., Kiesewetter, H. and Wenzel, E. (1990) Effect of Ginkgo biloba on fluidity of blood and peripheral microcirculation in volunteers. Arzneimittelforschung 40, 589-593.

Kim, Y. S., Pyo, M. K., Park, K. M., Park, P. H., Hahn, B. S., Wu, S. J. and Yun-Choi, H. S. (1998). Antiplatelet and antithrombotic effects of combination of ticlopidine and Ginkgo biloba extract (EGb 761). Thromb. Res. 91, 33-38.

Kimura, Y., Tani, T., Kanbe, T. and Watanabe, K. (1985). Effect of cilostazol on platelet aggregation and experimental thrombosis. Arzneimittelforschung 35, 1144-1149.

Nirogi, R. V., Kandikere, V. N., Shukla, M., Mudigonda, K., Shrivasthava, W., Datla, P. V. and Yerramilli, A. (2006). Simultaneous quantification of cilostazol and its primary metabolite 3,4-dehydrocilostazol in human plasma by rapid liquid chromatography/tandem mass spectrometry. Anal. Bioanal. Chem. 384, 780-790.

Oberpichler, H., Sauer, D., Rossberg, C., Mennel, H. D. and Krieglstein, J. (1990). PAF antagonist ginkgolide B reduces postischemic neuronal damage in rat brain hippocampus. $J$. Cereb. Blood Flow Metab. 10, 133-135.

Sastre, J., Millán, A., García de la Asunción, J., Plá, R., Juan, 
G., Pallardó, F. V., O'Connor, E., Martin, J. A., Droy-Lefaix, M. T. and Viña, J. A. (1998). Ginkgo biloba extract (EGb 761) prevents mitochondrial aging by protecting against oxidative stress. Free Radic. Biol. Med. 24, 298-304.

Suri, A. and Bramer, S. L. (1999). Effect of omeprazole on the metabolism of cilostazol. Clin. Pharmacokinet. 37(Suppl 2), 53-59.

Suri, A., Forbes, W. P. and Bramer, S. L. (1999). Effects of CYP3A inhibition on the metabolism of cilostazol. Clin. Pharmacokinet. 37(Suppl 2), 61-68.

Tucker, G. T., Houston, J. B. and Huang, S. M. (2001). Optimi- zing drug development: strategies to assess drug metabolism/transporter interaction potential-towards a consensus. Br. J. Clin. Pharmacol. 52, 107-117.

von Moltke, L. L., Weemhoff, J. L., Bedir, E., Khan, I. A., Harmatz, J. S., Goldman, P. and Greenblatt, D. J. (2004). Inhibition of human cytochromes P450 by components of Ginkgo biloba. J. Pharm. Pharmacol. 56, 1039-1044.

Zou, L., Harkey, M. R. and Henderson, G. L. (2002). Effects of herbal components on cDNA expressed cytochrome P450 enzyme catalytic activity. Life Sci. 71, 1579-1589. 